\title{
Yod
}

Revue des études hébraïques et juives

La littérature israélienne, miroir d'une société multiple

\section{À la recherche du Levant perdu : des écrivains d'Israël racontent l'Égypte}

The Search for the Lost time: Israeli Novelists Tell Egypt. (Three Israeli novelists and their memoirs)

אחר המזרח האבוד,מצרים בספרות הישראליתהחיפוש

\section{Rina Cohen Muller}

\section{OpenEdition}

Journals

\section{Édition électronique}

URL : https://journals.openedition.org/yod/362

DOI : $10.4000 /$ yod.362

ISSN : 2261-0200

Éditeur

INALCO

\section{Édition imprimée}

Date de publication : 1 octobre 2009

Pagination : 139-154

ISBN : 978-2-85831-178-1

ISSN : 0338-9316

\section{Référence électronique}

Rina Cohen Muller, «À la recherche du Levant perdu : des écrivains d'Israël racontent l'Égypte », Yod [En ligne], 14 | 2009, mis en ligne le 31 octobre 2011, consulté le 08 juillet 2021. URL : http:// journals.openedition.org/yod/362; DOI : https://doi.org/10.4000/yod.362

Ce document a été généré automatiquement le 8 juillet 2021.

\section{(c) (†) 8}

Yod est mis à disposition selon les termes de la Licence Creative Commons Attribution - Pas d'Utilisation Commerciale 4.0 International. 


\title{
À la recherche du Levant perdu : des écrivains d'Israël racontent l'Égypte
}

\author{
The Search for the Lost time: Israeli Novelists Tell Egypt. (Three Israeli novelists
} and their memoirs)

אחר המזרח האבוד,מצרים בספרות הישראליתהחיפוש

\section{Rina Cohen Muller}

1 Il est des périodes dans l'histoire d'une nation où la littérature entre en symbiose avec les mouvements de la société.

2 Il est un fait qu'une œuvre littéraire est un objet complet en soi. Cependant, son éclairage par l'espace humain où elle s'est composée et où elle s'exprime, lui donne une dimension supplémentaire. Celle de pouvoir comprendre l'influence qu'elle a pu avoir sur l'évolution de la société. Elle devient alors à la fois miroir et actrice d'une transformation multidimensionnelle de la manière de vivre et d'appréhender l'existence de l'autre. C'est donc d'un point de vue à la fois historique et sociologique qu'est abordée ici la littérature israélienne racontant l'Égypte.

Le processus d'intégration préconisé et mis en œuvre par le mouvement sioniste se fondait sur l'idée qu'il fallait donner naissance à un homme nouveau, faisant table rase de son passé - le sien ou celui de ses aînés - en diaspora.

Dans cette société, les immigrants juifs originaires des pays arabes et/ou musulmans sont considérés comme une masse indifférenciée, avec des dénominations comme 'edot ha-mizrah (les communautés de l'Orient), sfaradim, mizrahim (orientaux), puis yehudim 'aravim (juifs-arabes). Aucune d'entre elles ne correspond vraiment à la réalité. Elles dénotent soit un choix idéologique soit une conception par défaut. Si l'on a recours à l'une ou l'autre de ces expressions, on met dans un même panier tous les immigrants de ces pays en laissant entendre que leur histoire et leur manière de vivre sont les mêmes. Or nous nous trouvons en présence de cultures foncièrement différentes, issues des réalités vécues dans chacun des pays ${ }^{1}$.

5 La pratique d'intégration à marche forcée dans un moule unique et uniforme a volé en éclat. Il faut attendre la fin des années 1970 pour voir apparaitre un véritable courant 
d'œuvres ayant pour thème le pays d'origine, la rupture et le processus d'intégration dans le nouvel espace national. La nouveauté de ces écrits se situe dans le fait qu'ils racontent une histoire différente de celle évoquée jusque-là, consacrée tout entière à la longue souffrance des Juifs sous des régimes arabes. Le précurseur de ce phénomène novateur est un Juif d'Irak, Shimon Ballas avec son livre Ha-ma'bara («Le baraquement ») paru en $1964^{2}$.

6 Le premier roman israélien évoquant la vie de juifs en Égypte date de 1978, peu de mois après que le président Sadate a exprimé son souhait de se rendre à Jérusalem.

Du même coup, le plus grand ennemi d'Israël, l'Égypte, devient un ami. Ce bouleversement n'est certainement pas étranger à l'éclosion de cette nouvelle littérature dont la maturation est symbolisée par six ouvrages. Il s'agit de la trilogie de Yitzhak Goren Gormezano : Un été à Alexandrie ${ }^{3}$ (1978), Blanche $e^{4}$ (1986) et En route vers le $\operatorname{stade}^{5}$ (2003) ; de La mulukhiya de maman ${ }^{6}$ (2006) de Nissim Zohar et de l'œuvre la plus importante et la plus complète, celle de Ronit Matalon, Celui qui nous fait face ${ }^{7}$ (1995), le recueil d'essais Lire et écrire ${ }^{8}$ (2001) et Le son de nos pas ${ }^{9}(2008)^{10}$.

8 Le cadre historico-politique de ces récits est l'Égypte dominée par les Britanniques depuis 1882, celle du roi Farouk arrivé au pouvoir en 1936, du soulèvement en 1952 contre les Britanniques, du coup d'État du général Nagib en 1953, de la prise de pouvoir par Nasser en 1954 et pour finir, celle de la guerre du Sinaï en 1956.

On évalue le nombre de Juifs vivant en Égypte en 1948 entre 75.000 et 80.000 personnes. La communauté séfarade, dont la présence remonte à l'arrivée de Maimonide en Égypte (xII ${ }^{\mathrm{e}}$ siècle), s'agrandit régulièrement d'immigrants provenant principalement des cités méditerranéennes de l'Empire ottoman, avec une accélération au fur et à mesure que l'on avance dans le xix siècle avec le développement du commerce du coton et l'ouverture du canal de Suez (1869). Très rapidement les Séfarades vont constituer l'élite, une communauté à part et en même temps la plus importante en nombre.

10 La communauté ashkénaze, composée d'émigrants d'Europe de l'Est, se développe essentiellement à partir de la fin du XIX siècle, c'est-à-dire à l'époque de la première aliya. La plupart des Ashkénazes, comme des Séfarades, arrivés en Égypte au XIX siècle possèdent un passeport de l'une des grandes puissances européennes.

11 L'idée largement répandue selon laquelle la quasi-totalité des Juifs égyptiens a émigré en Israël est à nuancer, car les sources officielles avancent le chiffre de 35.000 personnes arrivées dans ce pays jusqu'en 1961. Des communautés importantes vivent aux États-Unis, au Canada, en France, en Italie et en Grande-Bretagne.

12 Les auteurs présentent des récits comparables qui couvrent une courte période allant des années 1940 au départ de leurs familles dans les années 1950. Un temps où le nationalisme arabe est exacerbé par la répression pratiquée par l'autorité militaire britannique locale. Le mouvement national dénonce le sionisme qu'il assimile au caractère colonial de cette tutelle. À cette époque aussi apparaît la haine des Juifs, en lien direct avec le conflit ouvert par la création de l'État d'Israël.

13 Les familles séfarades décrites dans les ouvrages s'étaient enrichies en profitant de l'économie coloniale britannique en Égypte, du commerce du coton, de la spéculation boursière et des paris sur les champs de courses. Leurs fortunes se sont quelque peu rétrécies, tout en laissant de quoi vivre dans une certaine aisance. Ce qui permet, encore pour un certain temps, de vivre dans l'illusion du «comme si » rien n'avait changé. Les loisirs occupent une grande place dans les récits, notamment chez Goren 
Gormezano et Zohar : les sorties, les bals, les parties de poker des hommes, le rendezvous hebdomadaire consacré aux parties de cartes entre femmes, appelé «matinée du rami ", etc. La vie se caractérise aussi par l'absence de toute pratique religieuse, voire par sa contestation. Les grandes fêtes religieuses sont néanmoins l'occasion de rencontres familiales.

Les récits décrivent ainsi une vie faite d'oisiveté, où le paraître est essentiel : « [comme] des comtes français, des consuls italiens, des effendis turcs et des officiers de l'armée de Sa Majesté Farouk ou George VI - tous avaient l'air d'être venus pour des vacances ${ }^{11}$. »

Il s'agit en quelque sorte de citoyens cosmopolites du monde colonial, ce que Goren Gormezano illustre dans Un été à Alexandrie :

"Vous ne pouvez pas comprendre comment des gens civilisés peuvent admettre un féodalisme colonial si attardé. Il est vrai qu'Alexandrie était pourrie, mais sa pourriture a de longues racines, chargées d'histoire [...]. C'est la raison pour laquelle je l'aime tant, cette Alexandrie. Une ville qui vous permettait de vivre comme un lord sans souci, sans être millionnaire. Il suffisait d'être juif ou européen et d'avoir un peu d'intelligence, mais même celle-ci n'était pas indispensable. L'argent? L'argent était destiné à être dépensé pour les plaisirs ou la débauche. [...] Des bals, des excursions, des croisières, des courses de chevaux et des parties de cartes. Vous gagnez entre 30 et 100 livres par mois. On paie 4,5 livres de loyer et on vit dans un palais, entouré de domestiques dont chacun gagne 2 livres par mois. Quel écart formidable! Et finalement vous n'êtes qu'un petit bourgeois minable ${ }^{12}$. »

Cette société juive est polyglotte : les langues parlées sont l'arabe, le judéo-espagnol, l'anglais, le français et souvent aussi la langue du pays d'origine de la famille.

L'essayiste Jacqueline Kahanoff explique :

"Dans ma jeunesse, il me semblait naturel que les habitants du Caire se comprennent entre eux-mêmes s'ils parlaient des langues différentes et portaient des noms d'origines différentes - musulmane, arabe, chrétienne, juive, syrienne, grecque, arménienne, italienne. Les noms des villes - Bagdad, Tunis, Alep, Beyrouth, Damas, Istanbul, Salonique, Casablanca, Jérusalem - étaient familiers, parce que les gens, surtout les proches, y faisaient des allersretours comme dans les pièces d'une unique grande maison ${ }^{13}$."

Et Ronit Matalon insiste dans Lire et écrire :

"Cette génération levantine nourrie par les différents univers culturels n'avait pas de langue maternelle [...]. Leur identité religieuse, comme leur identité nationale, était elle aussi une hybridation de choix multiples et d'imagination. Lorsqu'ils disaient "Allah", on ne savait pas si Allah était juif, musulman ou chrétien. Mais le plus important est que cela n'avait aucune importance pour eux ${ }^{14}$. "

19 Dans les récits de Zohar, Goren Gormezano et Matalon, la famille est gérée par les femmes, à l'image de ce que décrit Dina Tsvi Riklis dans son film Trois mères (2006) qui est d'abord une déclaration d'amour à une culture disparue. Les femmes, à l'instar de leurs sœurs musulmanes ou chrétiennes, sont confinées au foyer et n'en sortent qu'accompagnées par un membre masculin de la famille, même si ce n'est qu'un enfant. Les mariages sont des arrangements entre familles et ne peuvent être conclus qu'entre personnes d'une même communauté. Les rares épousailles qui dérogent à la règle sont vouées au désastre.

20 Très nettement, cette Égypte du deuxième tiers du siècle dernier décrite comme un paradis perdu rappelle la société urbaine ottomane de la fin du xix ${ }^{e}$ siècle - une société composée de communautés autonomes avec leurs règles particulières, dont les lieux de contact et de rencontre sont communs. En quelque sorte, « chacun connaît sa place » et "tous savent où et quand se rencontrer", car les codes de comportement de chacun sont connus de tous. "La société à Alexandrie étouffe dans la toile des convenances et 
donc toute allusion, la plus légère soit-elle, à une quelconque transgression libère des sentiments bien enfouis sous une couche de fallacieuses bienséances", dit Goren Gormezano dans Un été à Alexandrie ${ }^{15}$.

21 L'Alexandrie des Juifs devient ainsi un lieu mythique, la ville cosmopolite des bords de la Méditerranée. L'actualité politique n'y tient lieu que de toile de fond. En fait, on ne parle pas politique, les mots "sionisme», "socialisme» ou "communisme», sont parfois chuchotés, mais bannis des conversations mondaines comme des expressions honteuses ou malséantes, jusqu'au jour où la politique a tout fait exploser et a contraint les familles à partir.

Dans La mulukhiya de maman, Nissim Zohar raconte comment, à l'occasion du premier anniversaire de la mort de sa mère, il confectionne une mulukhiya, un plat qu'il n'a pas dégusté depuis qu'il a quitté l'Égypte environ cinquante ans plus tôt. Avec le récit minutieux de la confection du plat, la recherche des ingrédients, l'application méticuleuse de la recette allant jusqu'à reproduire les gestes qu'il a observés quotidiennement, l'auteur nous entraîne dans une véritable expédition où il nous fait découvrir l'histoire de ses relations complexes avec sa mère, le mode de vie de cette famille cairote aisée où tout se décide entre la mère et ses deux sœurs.

Dans sa trilogie, dont la trame est également une saga familiale se déroulant à la même époque à Alexandrie, Yitzhak Goren Gormezano met en lumière la fin d'un monde, sans que ses protagonistes n'en aient vraiment conscience, continuant à vivre " comme si de rien n'était». La nostalgie est ici l'instrument du travail de deuil face à la perte irrémédiable d'une manière de vivre.

À la différence de ces deux auteurs, Matalon centre son récit sur la rupture qui est en réalité déjà consommée avant même d'être ressentie. La nostalgie n'est déjà plus que mémoire. Tout ce qui reste, ce sont des photos de la famille avant son départ d'Égypte et après son éclatement dans plusieurs pays, dont Israël. Les clichés que l'on se dispute âprement sont une symbolique de l'héritage levantin que chacun des personnages possède, interprète à sa manière et veut garder pour lui seul. Cette nostalgie conduit à poser la question de la matérialité des racines et de l'identité.

Les différents protagonistes du roman quasi autobiographique de Matalon tentent, chacun à sa manière, d'y répondre en essayant d'y donner corps.

-L'oncle Cicourel s'efforce de perpétuer le caractère colonial de son mode de vie en s'installant au Cameroun où il a acquis en 1967 la luxueuse résidence d'un colon allemand.

- L'oncle Moïse, sioniste, se fixe dans un kibboutz qu'il quitte lorsqu'il prend conscience de la discrimination dont il est victime en sa qualité d'Oriental.

- L'oncle Édouard vit à Dimona ${ }^{16}$. Après avoir été un redoutable agent des services secrets israéliens à Gaza, il se réfugie dans un mode de vie arabe, aux dires de ses frères.

- L'oncle Henri Lehman, le mari français de tante Marcelle, lui-même rescapé de la Shoah, vit sur son passé de combattant de l'indépendance d'Israël et parcourt le monde dans une recherche frénétique de membres de sa famille. «La diaspora juive l'exaltait comme si dans la diaspora mondiale il avait l'opportunité de trouver des Lehman perdus, un élargissement possible de sa propre essence, des ambassadeurs éventuels de sa personnalité plantés au large par mégarde ${ }^{17}$. »

- La mère, Inès, plante et déplante des rosiers et ne comprend pas pourquoi ils ne prennent pas racine. Et, vers la fin du roman, alors qu'on lui demande si elle regrette d'avoir quitté l'Égypte, elle répond que non, mais qu'elle «s'en languit à en mourir » et, ajoute-t-elle, « notre vie là-bas a fini ». Zuza, sa nièce new-yorkaise, dit : «Vos racines sont là-bas [...]. 
Qu'avez-vous à chercher ici ?» Et Inès de répondre: "Racines, racines... foutaise. L'être humain n'a pas besoin de racines, il a besoin d'une maison ${ }^{18}$. » Gormezano :

Le départ d'Égypte et l'arrivée en Israël sont considérés comme une nécessité à laquelle il fallait se résigner. Dans En route vers le stade, Goren Gormezano la décrit ainsi :

"Mon père disait que s'il y avait un État pour les Juifs, il était normal que les juifs aillent y vivre. Tout simplement, non par engouement messianique, ni par enthousiasme sioniste. Tout simplement par la force de la logique. [...] Et par cette attitude, mon père a anticipé ce qu'il aurait été contraint de faire de toute façon et de cette manière, il a réussi à être maître de son destin ${ }^{19}$.»

Et si pour Zohar, "en secret, tout le monde parlait du jour où il faudrait quitter l'Égypte. Mais ce n'était pas encore le moment. Les Anglais gouvernaient encore. Et si on devait émigrer, pourquoi en Palestine ? Qu'y avait-il d'autre là-bas que la guerre, le sable et les travaux forcés? ». Il n'empêche que le jour du départ est arrivé et «les membres de la famille restants sont partis comme des réfugiés. Le sionisme était pour eux plus une bouée de sauvetage dans la tourmente de l'incertitude qu'une passion

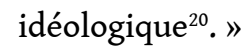

Le départ était considéré comme inévitable et donc, on tentait de se faire encore une raison malgré la souffrance de quitter un monde dont l'on savait, intimement, qu'il était révolu et destiné à disparaître, du fait même de sa manière de fonctionner.

L'arrivée en Israël est, en revanche, un véritable choc que Goren Gormezano illustre d'une manière inattendue :

«Dans les années 1960, nous sommes allés rendre visite à Raphaël à Beer-Sheva, dans un de ces blocs misérables qui ont poussé dans le désert. Ce ne sont pas la misère et l'état d'abandon des lieux qui nous ont choqués. Le choc a eu lieu lorsque nous avons demandé à Raphaël de chanter pour nous. Il a bégayé, puis il a dit qu'il avait perdu la voix ${ }^{21}$. "

Dans Lire et écrire, Matalon montre qu'en fait, cette incompréhension était logique, car elle était la conséquence d'une réciproque et totale ignorance : « Il n'y avait rien dans la société coloniale, multilingue, multinationale des immigrants [Égyptiens] pour les préparer à la rencontre avec une autre société d'immigrants, l'Israélienne, dont les règles du jeu étaient foncièrement différentes : non pas une culture à côté d'une autre, mais une culture à la place d'une autre, monolithique et cherchant à s'imposer ${ }^{22}$. »

Alors, on tente de résister, de faire une fois de plus comme si de rien n'était, dans une sorte d'ultime et dérisoire coup de panache au monde perdu, raconté par Goren

«Là-bas, en Égypte, il y avait une différence entre les gens du Caire et ceux d'Alexandrie, entre Séfarades et Ashkénazes. Mais dans la ma'bara tous se réunissaient pour les parties de cartes et des "partys", des bals qu'ils organisaient tous les vendredis soirs. [...] Il y avait quelque chose de formidable dans cet entêtement des Égyptiens à poursuivre la vie dans la ma'bara exactement comme au Caire et à Alexandrie. Ils ont tout simplement ignoré les difficultés avec élégance. [...] Il fallait les voir sortir, surtout en hiver, habillés élégamment, lui en veston cravate, elle en robe de taffetas bien décolletée à la mode des années 50, portant des bottes en caoutchouc, traversant l'allée dans la gadoue, tenant à la main leurs chaussures vernies... ${ }^{23}$ "

Mais il n'y a pas d'autre issue : c'est ici qu'il faut désormais vivre. Il n'est pas possible de revenir en arrière, de retourner au temps d'Alexandrie. Alors reste le rêve du paradis perdu. La mère de Zohar refuse de s'y laisser prendre : « Ce qui est passé est mort », ditelle ${ }^{24}$ et tante Victorine renchérit : «Le passé est mort et ne va pas ressusciter, et si on regarde en arrière on ne voit pas ce qu'il y a devant soi. » Mais, s'étonne Zohar, «tout le 
monde réfléchit au passé, non?", à quoi tante Victorine répond : «Pour un instant seulement et pas plus, autrement on se casse la figure ${ }^{25}$. »

La fascination du paradis perdu constitue parfois un nœud, difficile à défaire, serré par la peur de ne pas retrouver dans la réalité l'image qu'on s'était peu à peu dessinée. Car plus la représentation se densifie avec le temps - au point de devenir un semblant de réalité - plus on rêve de la voir se matérialiser tout en sachant qu'au fond, son existence s'estompe au fil du temps. La mère de Matalon, invitée par sa nièce à faire un voyage en Égypte, refuse dans un premier temps de rompre le nœud : «Il n'y a aucune raison de partir. Et pour voir quoi ? Les gens que j'ai aimés sont morts ou partis, et que vaut encore un lieu sans $\operatorname{ses}^{26}$ ?» Mais, finalement, elle se laisse convaincre et elle revient transfigurée d'Égypte, « avec un nouveau ressort : l'émotion d'une joie retenue qui rayonnait autour d'elle sur la pointe des pieds. Quelque chose a surgi du bitume collant qui recouvrait sa vie précédente et qui est revenu sous une autre forme, purifié par le temps, innocent comme du pain ${ }^{27}$.»

Une manière d'être, faite de douceur et enracinée dans le monde de la Méditerranée orientale, peut-elle fleurir dans une communauté nationale dont les prescripteurs, tout en se targuant d'édifier un foyer fusionnel, s'accrochent farouchement à son occidentalo-centrisme, comme si la vigueur de la nation dépendait de la nature « occidentale» de sa culture?

Dans le même temps Matalon prévient :

"Si mes propos laissent entendre une sorte d'attirance affective pour cet " autre " monde, le supposé utopique duquel viennent mes parents et leurs congénères, il faut la réfuter tout de suite: Le Caire du régime colonial était loin d'être une utopie, certainement pas pour ceux qui y vivaient et à qui elle appartenait - les musulmans. »

Mais ce qui la désole « est le sentiment de gaspillage de la possibilité culturelle ratée dans la société israélienne d'immigrants, une proposition sociale et culturelle qui est restée finalement muette et n'a pas tracé sa voie ${ }^{28}$. 》

Et c'est là que la nostalgie des parents de Gormezano, les regrets de la mère de Zohar et la métamorphose de celle de Matalon se complètent : il s'agit effectivement de ne pas arracher la page du passé levantin, mais de la tourner afin de pouvoir la relire à tout moment pour construire une autre histoire (nationale) rejetant, comme la romancière, toute catégorisation: récit de femme, récit oriental, israélien, occidental, une transcendance qui du coup remet toutes ces étiquettes en question. Et comme l'explique Hanan Hever, ce jeu des identités constitue une identitée ${ }^{9}$.

Mais la réflexion va encore plus loin, au-delà de la finitude d'une imaginaire immuabilité de la société dans laquelle on vit.
"Il me semble que l'un des biens les plus précieux que les gens de la génération levantine ont apporté est la conscience de la relativité culturelle qui était ancrée en eux, non pas comme une idéologie déclarée, mais comme partie organique de leur mode de vie et de leur nature, tout comme la couleur des yeux et le ton de la voix. [Ils ont apporté] cette conscience qu'une identité est quelque chose de relatif et non pas total, qu'elle ne peut être "hors contexte", qu'elle est en relation de réciprocité permanente avec l'environnement et la société, qu'elle change ou bien qu'elle a la capacité de se transformer. Cette conscience était en confrontation, voire en opposition, avec le narratif sioniste, qui a prôné une seule identité nationale, nouvelle, enracinée ${ }^{30}$."

On peut aujourd'hui envisager de pouvoir édifier une identité hétérogène et mouvante, en transformation. On se pose de moins en moins la question de l'identité sous l'angle de l'alternative entre l'Occident et l'Orient, mais on parle de plus en plus d'alternative 
méditerranéenne. Celle-ci ne pourra se fonder sur l'hypothèse qui fige les protagonistes, Juifs et Arabes, ou - autres illusions dangereuses - Occident et Orient, ou encore Europe et Méditerranée. Elle peut devenir réelle et vivante si elle s'enracine dans l'idée qu'entre les peuples vivant dans le bassin méditerranéen, existent une proximité et un tissu de sensibilités géoculturelles qui permettent d'initier le dialogue dans cet espace. À condition que ce dialogue ne se fonde pas sur des rapports de domination comme l'affirme Jacqueline Kahanoff :

"Je suis une Levantine typique dans le sens où j'ai une égale estime pour ce que j'ai reçu de mes origines orientales et pour ce que j'ai acquis de la culture occidentale. Dans cette fécondité réciproque, qu'en Israël on appelle avec condescendance levantinisation, je vois un enrichissement et non une dépréciation. Le levantinisme est la seule voie pouvant conduire un lien possible entre nous et nos voisins. Si nous n'avons pas peur de la levantinisation, nous édifierons une route pour la paix où nombreux seront ceux qui pourront coopérer d'une manière concrète, ou du moins faire preuve de plus de tolérance ${ }^{31}$. "

\section{Quelques précisions sur les auteurs et les œuvres}

\section{Nissim Zohar}

(Homme de théâtre né à Alexandrie en 1937, a immigré en Israël en 1950.)

La mulukhiya de maman (המולוכייה של אמא), Tel-Aviv, Yedioth Ahronoth, 2006

Après le décès de sa mère à Bat-Yam, l'auteur décide de préparer le plat populaire égyptien, la mulukhiya. La recherche des ingrédients et de la recette le renvoie à son enfance au Caire. Élevé par sa mère, il se sent coupable depuis son enfance d'avoir survécu à son frère, mort dans un accident de la circulation et croit que sa mère aurait souhaité qu'il meure à la place de son frère. Ce voyage dans le passé lui permet de se réconcilier avec lui-même. À travers ce récit, il trace la vie quotidienne d'une famille aisée à l'époque du roi Farouk.

\section{Yizhak Goren Gormezano}

(Homme de théâtre, rédacteur en chef de la revue Ha-kiwun mizrah et écrivain né à Alexandrie en 1941 a immigré en Israël en 1951.)

\section{Un été à Alexandrie (קיץ אלכסנדרוני), Tel-Aviv, Am Oved, 1978}

41 La famille de Robi vit dans le quartier cossu de Sporting-Club à Alexandrie. Tous les étés, elle loue des chambres à ses proches, la famille Hamdi-Ali, qui vient pour participer aux courses de chevaux qui s'y déroulent. Joseph Hamdi Ali qui a fait fortune dans le milieu hippique a imposé le métier de jockey à son fils. Tout l'été, la vie des deux familles tourne autour des courses et des loisirs. L'échec du jockey est perçu par son père comme une punition d'Allah en raison de sa conversion au judaïsme pour l'amour d'Émilie.

\section{Blanche (בלאנש), Tel-Aviv, Am Oved, 1989}

Blanche, âgée de 18 ans, ne supporte pas sa condition à Alexandrie à la fin des années 1940 où « il était admis que les Arabes étaient pauvres et les Juifs et les Européens, 
riches. " Elle cherche à faire un beau mariage, mais découvre que les conventions sociales l'en empêchent. Sa grand-mère lui arrange un mariage avec Raphaël, beau, mais désargenté. Ce dernier, sans ambition véritable, se laisse séduire par le communisme puis par le sionisme. Il fait partie d'un petit groupe qui cherche à organiser une cellule sioniste à Alexandrie. Ne rencontrant aucun succès auprès des Juifs d'Alexandrie, il va à Tel-Aviv pour chercher des appuis. On ne le prend pas au sérieux. Blanche et Raphaël s'installent en 1950 à Beer-Sheva où ils divorcent.

\section{En route vers le stade (בדרך לאצטדיון), Tel-Aviv, Kedem, 2003}

L'auteur veut rendre hommage à son père décédé avant d'avoir pu publier son autobiographie et dont les notes remplissent sept cahiers. Près de 40 ans plus tard, le fils décide d'écrire cette biographie. Mais sa propre histoire prend le dessus sur celle du père. Robi (l'auteur) devenu Yitzhak en fait la dernière partie de la trilogie, en reprenant l'histoire de la famille à Alexandrie jusqu'à nos jours en Israël.

\section{Ronit Matalon}

(Écrivain et essayiste née en 1959 à Gane-Tiqva dans la banlieue de Petah-Tiqva)

\section{Celui qui nous fait face (זה עם הפנים אלינו), Tel-Aviv, Am Oved, 1995}

Esther, âgée de 17 ans, rend visite à son oncle au Cameroun où elle pourrait éventuellement s'installer. Elle nous livre un double récit, celui de son séjour à Douala, consigné dans son journal intime, et celui de l'histoire de sa famille éclatée avant d'avoir quitté Le Caire. On découvre ainsi l'errance de chacun des membres de la famille.

\section{Le son de nos pas (קול צעדינו), Tel-Aviv, Am Oved, 2008}

La vie de l'auteure et celle de sa mère dans une baraque de la ma'bara de Gane Tiqva sont au cœur du récit. Lucette élève ses trois enfants en gagnant péniblement de quoi vivre comme femme de ménage. Le père les a abandonnés en s'engageant dans une forme d'agitation politique.

\section{BIBLIOGRAPHIE}

HEVER, Hanan (2007), Ha-sippur ve-ha-leom, Resling, Tel-Aviv.

KAHANOFF, Jacqueline (2005), Ben Shne 'olamot, Keter, Jerusalem.

SHIM'ONI, Batya (2008), 'Al saf ha-geula, sippur ha-ma'abara: dor rishon ve-sheni, Tel-Aviv, Kinneret, Zmora-Bitan, Dvir. 


\section{NOTES}

1. On peut observer incidemment qu'il s'agit là de la véritable trame de ce que l'on appelle siah 'adati (le débat communautaire) qui se poursuit aujourd'hui encore en Israël.

2. Batya Shim'oni, 'Al saf ha-ge'ulla, sippur ha-ma'bara: dor rishon we-sheni, Tel-Aviv, Kinneret, Zmora-Bitan, Dvir, 2008, p. 32.

3. Qayits aleksandroni, Tel-Aviv, Am Oved.

4. Tel-Aviv, Am Oved.

5. Ba-derekh la-itstadyon, Tel-Aviv, Kedem.

6. Ha-mulukhiya shel imma, Tel-Aviv, Yedioth Ahronoth.

7. Ze 'im ha-panim elenu, Tel-Aviv, Am Oved.

8. Qro u-khtov, Tel-Aviv, Sifriat Poalim

9. Qol tse 'adenu, Tel-Aviv, Am Oved.

10. Aucune de ces œuvres n'a été publiée en français. La traduction des extraits cités ici est de l'auteur de l'article.

11. Y. Goren Gormezano, Un été à Alexandrie, p. 56.

12. Ibid. p. 15.

13. Jacqueline Kahanoff, Ben shne 'olamot, Jérusalem, Keter, 2005, p. 49.

14. R. Matalon, Lire et écrire, p. 45.

15. Ibid., p. 17.

16. Ville fondée en 1955, à $36 \mathrm{~km}$ au sud-est de Beer-Sheva en plein désert, habitée essentiellement par des Juifs d'Afrique du Nord et, depuis les années 1990, par des immigrants russes.

17. R. Matalon, Celui qui nous fait face, p. 168.

18. Ibid., pp. 294-295.

19. Y. Goren Gormezano, En route vers le stade, p. 73.

20. N. Zohar, La mulukhiya de maman, p. 155.

21. Y. Goren Gormezano, Blanche, p. 180.

22. R. Matalon, Lire et écrire, p. 45.

23. Y. Goren Gormezano, En route vers le stade, p. 110.

24. N. Zohar, La mulukhiya de maman, p. 13.

25. Ibid. p. 68.

26. R. Matalon, Le son de nos pas, p. 410.

27. Ibid. p. 413.

28. R. Matalon, Lire et écrire, p. 45.

29. Hanan Hever, Ha-sippur we-ha-le'om, Tel-Aviv, Resling, 2007, p. 333.

30. R. Matalon, Lire et écrire, p. 45.

31. « La première dame de Méditerranité », Maariv, 15 mars 1996.

\section{RÉSUMÉS}

Romanciers israéliens originaires d'Égypte. De nombreux romans écrits par des Israéliens d'origine égyptienne - Nisim Zohar, Itzhak Goren-Gormezano et Ronit Matalon - ont été publiés depuis la fin des années 70. Ils racontent tous des histoires autobiographiques similaires : la vie simple et confortable en Égypte dans les années 40-50, le départ, la complexe d'intégration dans 
le melting pot israélien et la dislocation des familles. Tous ces récits se concentrent sur la mémoire et l'identité. Pour Zohar, c'est une question de souvenirs, pour Goren-Gormezano, il s'agit de nostalgie. Pour Matalon, le problème est la formation d'une identité israélienne, qui n'éradiquerait pas le passé égyptien, mais l'intégrerait dans une nouvelle construction, allant dans le sens d'une entente moyen-orientale, basée sur la vision d'une durable culture de tolérance dans la région.

Novels, written by Israeli of Egyptian origin - Nisim Zohar, Itzhak Goren-Gormezano and Ronit Matalon - have been published since the late 1970's. They all tell similar autobiographical stories: the easy and comfortable life in Egypt during the 1940-50's, the departure, the complex integration in the Israeli melting pot and the dislocation of the families. All these narratives are focused on memory and identity. For Zohar it is a question of remembrance, for GorenGormezano it is nostalgia and mourning for the past. For Matalon the issue is the formation of an Israeli identity, that does not eradicate the Egyptian past but integrates it in a new construction, going towards a Near-Eastern understanding, based on the vision of the long lasting tolerance culture in that area.

מאז 1970 יצאו לאור בישראל רומנים שחוברו על ידי סופרים יוצאי מצרים: ניסים זוהר, יצחק גורן

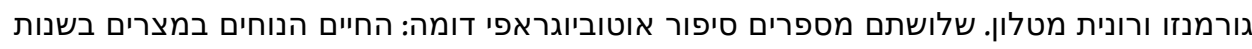

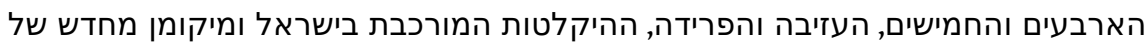

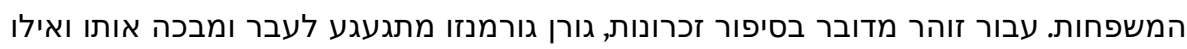

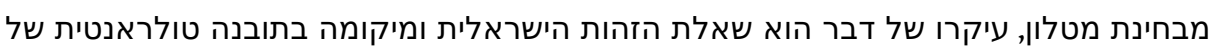
המזרח התיכון.

\section{INDEX}

Mots-clés : Goren-Gormezano Itzhak, juifs d'Égypte, Nisim Zohar, Ronit Matalon

Keywords : Zohar Nisim, Goren-Gormezano Itzhak (1941-), Matalon Ronit (1959-), Israel, literature, twentieth century, Israeli society, quest for identity, Sephardic, Jews from Egypt, Egypt

Index chronologique : vingtième siècle

Thèmes : littérature

\section{מילות מפתח}

הספרדי, יהודי מצרים, ניסים זוהר, רונית מטלון, השתלבות, הוצאה מן הכלל, ישראל,: ספרות, המאה העשרים, החברה הישראלית, החיפוש אחר הזהות, מצרים

Index géographique : Israël, Égypte 